\title{
Biomechanics Potential of the Masseter and Temporal Muscles in the Mandibles of Mesofacial Subjects
}

\author{
1 Department of Nursing and Odontology, Universidade de Santa \\ Cruz do Sul, Santa Cruz do Sul, RS, Brazil \\ 2 Department of Biology and Pharmacy, Universidade de Santa Cruz \\ do Sul, Santa Cruz do Sul, RS, Brazil \\ 3 Department of Morphological Sciences, Instituto de Ciências Básicas da \\ Saúde, Universidade Federal do Rio Grande do Sul, Porto Alegre, RS, Brazil \\ 4 Postgraduation in Neuroscience Program, Instituto de Ciências Básicas da \\ Saúde, Universidade Federal do Rio Grande do Sul, Porto Alegre, RS, Brazil \\ 5 Department of Basic Health Sciences, Universidade Federal de \\ Ciências da Saúde de Porto Alegre, Porto Alegre, RS, Brazil \\ 6 Department of Physical Education and Health, Postgraduate \\ Program in Health Promotion, Universidade de Santa Cruz do Sul, \\ Santa Cruz do Sul, RS, Brazil \\ J Morphol Sci 2018;35:180-182.
}

Ana Paula Chitolina Pradebon ${ }^{1}$ Marieli Chitolina Pradebon ${ }^{1}$ Guilherme Reghelin Goulart ${ }^{2}$ Geraldo Pereira Jotz ${ }^{3}$ Tais Malysz $z^{3,4}$ Júlia Niero Páfaro ${ }^{5}$ Magda de Souza Reis ${ }^{1}$ Deivis de Campos $2,5,6$

Address for correspondence Deivis de Campos, PhD, Departamento de Ciências Básicas da Saúde, Universidade Federal de Ciências da Saúde de Porto Alegre - UFCSPA, Avenida Sarmento Leite 245, 90050170, Porto Alegre, RS, Brazil (e-mail: dcampos@ufcspa.edu.br).

\begin{abstract}
Introduction The knowledge of the organization of the masseter muscle (MM) and the temporal muscle (TM) is extremely important when related to the study of the stomatognathic system. Moreover, some authors have shown that mastication is of great importance, not only for the intake of food but also for the systemic, mental and physical functions of the body.

Materials and Methods We have decided to analyze the biomechanical potential (length of the force arm, muscular work and mechanical advantage) of the MM and TM in the mandibles of mesofacial subjects $(n=34)$.

\section{Keywords}

- masseter muscle

- temporal muscle

- mesofacial

- mandibles

Results Our results show that the MM exhibits a better biomechanical potential than the TM $(p=0.0001)$.

Conclusion With these data, orthodontists may develop a specific treatment plan and get better results, especially in cases of patients in whom the biomechanical pattern of the temporomandibular joint is unfavorable.
\end{abstract}

\section{Introduction}

Some studies describe the type of fiber, the thickness and the neuromuscular pattern of the masticatory muscles. ${ }^{1-5}$ However, the descriptions regarding the biomechanical organization of the masticatory muscles in specific craniofacial standards are still inadequate, especially those that aim to elucidate the biomechanical differences between the human masseter muscle (MM) and the temporal muscle (TM).

Knowledge of the organization of the MM and of the TM is extremely important when related to the study of the stomatognathic system. This knowledge of the masticatory muscle organization assists in researches associated with occlusion, facial growth and temporomandibular disorders. ${ }^{6-8}$ Thus, we have decided to analyze the biomechanical potential (length of the force arm, muscular work and mechanical advantage) of the MM and of the TM in the mandibles of mesofacial (ME) subjects.

For the present study, we have used 34 mandibles of adult subjects (mean age $=45.5 \pm 7.1$ years old) of both genders, from the collection of the Laboratory of Human Anatomy of the Universidade de Santa Cruz do Sul (UNISC, in the Portuguese acronym) (ethics committee-protocol 141346). All of the quantification procedures of the length of the force arm, received

September 16, 2017 accepted

September 3, 2018

published online

November 14, 2018
DOI https://doi.org/

10.1055/s-0038-1675569. ISSN 2177-0298.
Copyright $\odot 2018$ by Thieme Revinter

Publicações Ltda, Rio de Janeiro, Brazil
License terms

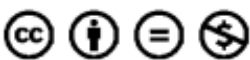


muscular work and mechanical advantage were made according to previous protocols. ${ }^{9}$

The distance between the condylar process and the coronoid process (the insertion site of the temporal muscle) represents the length of the force arm (LFA) of the TM, whereas the distance between the condylar process and the mental protuberance is the length of the resistance arm (LRA) (-Fig. 1). Similarly, the distance between the condylar process and the anterior border of the masseteric tuberosity (the insertion site of the masseter muscle) represents the LFA of the MM, whereas the distance between the condylar process and the mental protuberance is the LRA (-Fig. 1). Thus, the mechanical advantage of the TM and of the MM can be obtained using the following ratio: LFA/LRA. The inverse of this ratio represents the muscular work (LRA/LFA) of both muscles.

All of the measurements were performed on both sides (right and left) of all mandibles. However, we have decided to use data from one side (left) only because, using the paired ttest, we have concluded that there is no statistical difference between the sides and no apparent tendency toward any such difference $(p=0.3574)$. Only mandibles without any deformity or evidence of bone pathology were used. A $p$-value $\leq 0.05$ was considered significant in all statistical tests performed. Data were analyzed with the aid of the GraphPad Prism 5.01 software (GraphPad Software, Inc., San Diego, CA, USA).

In the present study, the comparison (paired t-test) of the biomechanical potential between the MM and the TM in the mandibles of ME subjects showed that the results were significantly higher in MM for LFA and mechanical advantage
Table 1 Comparison of all morphometric parameters estimated

\begin{tabular}{|l|l|l|l|}
\hline $\begin{array}{l}\text { Morphometric } \\
\text { Parameters }\end{array}$ & $\begin{array}{l}\text { Masseter } \\
\text { Muscle } \\
\text { Mean } \pm \text { SD }\end{array}$ & $\begin{array}{l}\text { Temporal } \\
\text { Muscle } \\
\text { Mean } \pm \text { SD }\end{array}$ & $p$-value \\
\hline $\begin{array}{l}\text { Length of the } \\
\text { resistance } \\
\text { arm }-L_{\text {RA }} \\
(\mathrm{mm})\end{array}$ & $106.14 \pm 5.82$ & $106.14 \pm 5.82$ & - \\
\hline $\begin{array}{l}\text { Length of the } \\
\text { force arm - } \\
\text { (mm) }\end{array}$ & $44.6 \pm 4.7$ & $36.9 \pm 3.0$ & $\mathbf{0 . 0 0 0 1}$ \\
\hline $\begin{array}{l}\text { Muscular work } \\
\left(\mathrm{L}_{\mathrm{RA}} / \mathrm{L}_{\mathrm{FA}}\right)\end{array}$ & $2.39 \pm 0.21$ & $2.88 \pm 0.23$ & $\mathbf{0 . 0 0 0 1}$ \\
\hline $\begin{array}{l}\text { Mechanical } \\
\text { advantage } \\
\left(\mathrm{L}_{\mathrm{FA}} / \mathrm{L}_{\mathrm{RA}}\right)\end{array}$ & $0.42 \pm 0.03$ & $0.34 \pm 0.02$ & $\mathbf{0 . 0 0 0 1}$ \\
\hline
\end{tabular}

Abbreviations: SD, standard deviation.

(17.2\% and $19.0 \%$, respectively). Thus, the muscular work of the TM proved to be $17.0 \%$ higher than that of the MM (-Table 1).

Finally, the present work aims to provide a simple but multidisciplinary synthesis of the current knowledge concerning the morphogenesis of the biomechanical organization of the main muscles of mastication and to help to promote future studies in this area. With these data, orthodontists may develop a specific treatment plan and get better results, especially in cases of patients whose biomechanical pattern of the temporomandibular joint is unfavorable.

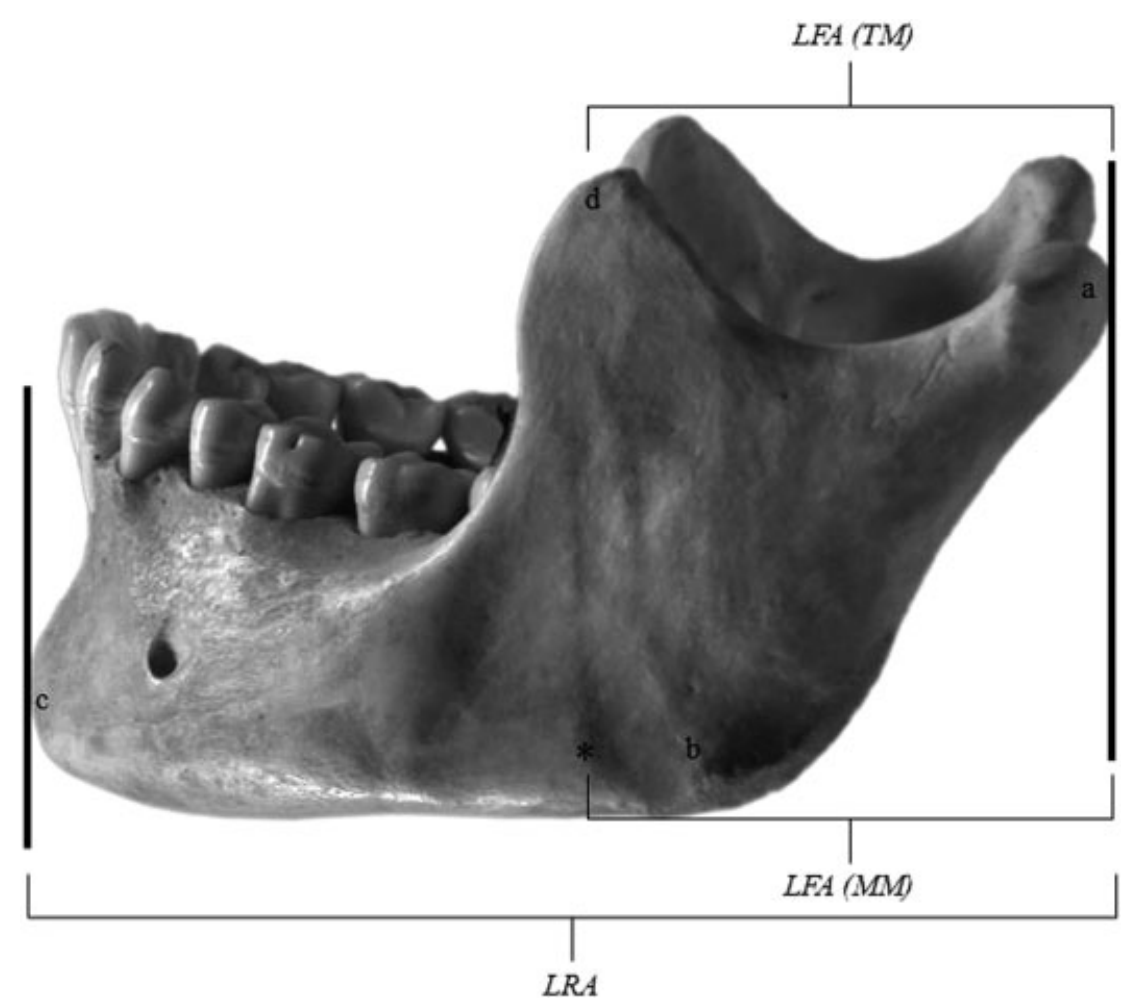

Fig. 1 Schematic drawing of the mandible showing the specific points that were used to measure the length of the force arm (LFA) and the length of the resistance arm (LRA) of the masseter muscle (MM) and temporal muscle (TM). a, condylar process; b, masseteric tuberosity; *, anterior border of the masseteric tuberosity; c, mental protuberance. 
182 Biomechanics of the Masseter and Temporal Muscles Pradebon et al.

Therefore, the purpose of the present study is to compile the latest scientific information concerning the relationship between mastication and general health.

\section{Conflicts of interest}

The authors have no conflicts of interest to declare.

\section{References}

1 Boer NP, Batigália F, Alonso LG, Ribeiro EC. Classification methods for human masticatory muscle fibers: critical literature review. Braz J Morphol Sci 2007;24(03):164-169

2 Emshoff R, Emshoff I, Rudisch A, Bertram S. Reliability and temporal variation of masseter muscle thickness measurements utilizing ultrasonography. J Oral Rehabil 2003;30(12):1168-1172

3 Farella M, Bakke M, Michelotti A, Rapuano A, Martina R. Masseter thickness, endurance and exercise-induced pain in subjects with different vertical craniofacial morphology. Eur J Oral Sci 2003;111 (03):183-188
4 Korfage JA, Brugman P, Van Eijden TM. Intermuscular and intramuscular differences in myosin heavy chain composition of the human masticatory muscles. J Neurol Sci 2000;178(02):95-106

5 Palinkas M, Nassar MS, Cecílio FA, et al. Age and gender influence on maximal bite force and masticatory muscles thickness. Arch Oral Biol 2010;55(10):797-802

6 Ariji Y, Sakuma S, Izumi M, et al. Ultrasonographic features of the masseter muscle in female patients with temporomandibular disorder associated with myofascial pain. Oral Surg Oral Med Oral Pathol Oral Radiol Endod 2004;98(03):337-341

7 Bakke M, Thomsen CE, Vilmann A, Soneda K, Farella M, Møller E. Ultrasonographic assessment of the swelling of the human masseter muscle after static and dynamic activity. Arch Oral Biol 1996; 41(02):133-140

8 Raadsheer MC, Kiliaridis S, Van Eijden TM, Van Ginkel FC, PrahlAndersen B. Masseter muscle thickness in growing individuals and its relation to facial morphology. Arch Oral Biol 1996;41(04):323-332

9 Marques HB, Richter FF, Heck L, Xavier LL, de Campos D. Biomechanical potential of the temporal muscle in brachyfacial and dolichofacial subjects: a study on dry mandibles. Orthod Craniofac Res 2016;19(03):162-168 